\title{
Review of: "Genetic surveillance for monitoring the impact of drug use on Plasmodium falciparum populations"
}

Kiswendsida Thierry Guiguemde

Potential competing interests: The author(s) declared that no potential competing interests exist.

An original article that draws attention to the use of drugs that may be a risk of the appearance of resistant strains of parasites. 